\title{
Methodenausbildung im digitalen Zeitalter: Neue Daten, Projektseminare und Selbstlernmodule
}

\author{
Holger Döring (D) Phillip Hocks $\mathbb{D}$
}

Online publiziert: 25. November 2020

(C) Der/die Autor(en) 2020

Zusammenfassung Die Datengrundlage in der Politikwissenschaft hat sich in den letzten Jahren stark verändert und ist breiter geworden. Daten sozialer Medien und digitale Spurendaten erweitern das Methodenportfolio in den Sozialwissenschaften beträchtlich. In diesem Artikel diskutieren wir Herausforderungen und Chancen dieser neuen Entwicklungen für die quantitative Methodenlehre in der Politikwissenschaft. Wir argumentieren, dass die Methodenlehre (i) stärker an Theorie und Inhalt geknüpft werden sollte. Dies erfolgt am besten durch (ii) Projektseminare, welche Studierende eigene Forschungsvorhaben umsetzen lassen. Mit der leichten Generierung durch neue Datenquellen, können Studierende (iii) den gesamten Forschungsprozess durchlaufen. Geringere Teile der Lehre werden als Präsenzveranstaltung abgehalten, (iv) Programmierfähigkeiten werden durch E-Lernangebote ergänzt. Durch die hohe Praxisorientierung und eigene Lerntempi kann so ein Interesse für quantitative Methoden bei mehr Studierenden der Politikwissenschaft gebildet werden.

Schlüsselwörter Quantitative Methodenlehre · E-Lernen · Selbstlernmodule · Projektseminare

\footnotetext{
H. Döring $(\triangle)$

Fachbereich Sozialwissenschaften, Universität Bremen, Postfach 330 440, 28359 Bremen, Deutschland

E-Mail: doering@uni-bremen.de

P. Hocks

Institut für Politikwissenschaft, Universität Münster, Scharnhorststraße 100, 48151 Münster,

Deutschland

E-Mail: phillip.hocks@uni-muenster.de
} 


\title{
Teaching Methods in the Digital Era: New Data, Project Seminars, and Self-learning Modules
}

\begin{abstract}
In recent years, data structures in political science have changed and are now more diverse. Social media and digital trace data broaden the set of sources and the methodological portfolio in the social sciences. Here, we review and summarize these recent developments in the context of teaching political science methods. We argue that (i) teaching methods should be more connected to theoretical and substantive questions. Further, (ii) this should take place in the format of project seminars, which allows students to conduct independent research. Through new data sources, (iii) students are able to follow the entire research process - from generating data to analyzing the information. Only parts of the learning will take place in typical classroom teaching; (iv) programming skills will be supplemented through e-learning elements. The high focus on active learning elements and individual learning speed should raise the interest for quantitative methods among students of political science.
\end{abstract}

Keywords Teaching - Quantitative methods · E-learning - Self-learning modules · Project seminars

\section{Einleitung}

Die Vermittlung von quantitativen Methoden ist heute ein etablierter und wichtiger Bestandteil des sozialwissenschaftlichen Studiums. Die Fähigkeit zur Anwendung dieser Methoden am Ende des Studiums der Politikwissenschaft variiert jedoch enorm innerhalb von Studiengängen und zwischen Standorten in Deutschland. Nutzen die einen bereits neue Analyseverfahren, um zum Beispiel große Datenmengen sozialer Medien auszuwerten, so haben viele andere Studierende ihr Wissen zu quantitativen Verfahren nach der Klausur schnell vergessen und nie angewendet. Es mangelt häufig an einer systematischen Integration quantitativer Methoden in der Breite des Studiums und an der Einbindung neuer Datenquellen.

Studierende sind daher nicht ausreichend auf die Herausforderungen neuer Datenmengen und der Digitalisierung vorbereitet. In der Politikwissenschaft und in den Berufsfeldern der Absolventen unserer Studiengänge sind die Breite, die Bedeutung und das Auswertungspotenzial von Daten enorm gestiegen. Diese neuen Datenquellen und Analyseverfahren verlangen auch eine Neujustierung der Methodenausbildung (Munzert 2014).

Wir gehen in unserem Beitrag auf didaktische und technische Herausforderungen von Datenwissenschaft (Data Science) in der universitären Lehre ein. Didaktisch gilt es, die Vermittlung von Methodenkenntnissen stärker durch eine fachliche Einbindung in Projektseminaren zu verzahnen. Dabei können umfangreich E-Lernmaterialien eingebunden werden. Aus technischer Perspektive sollten Studierende die Fähigkeit zur Analyse neuer Datenquellen und grundlegende Programmierfähigkeiten erwerben können.

Mit unserem Beitrag wollen wir einen Denkanstoß geben und Umsetzungsbeispiele für die Erweiterung der Methodenlehre an Hochschulen präsentieren. Un- 
sere Perspektive auf Methodenlehre umfasst dabei drei Aspekte: das allgemeine Forschungsdesign, Statistik und Programmierfähigkeiten. Wir konzentrieren uns in dem Beitrag auf Lehrkonzepte für quantitative Methoden, welche durch die umfangreiche Verbreiterung digitaler Daten vor neuen Herausforderungen stehen. In einem ersten Teil skizzieren wir dafür zunächst die aktuellen Veränderungen ${ }^{1}$ und Herausforderungen. In einem zweiten Teil diskutieren wir Stand und Defizite der Methodenausbildung. In einem dritten und letzten Teil präsentieren wir didaktische und technische Ansätze für eine Verbreiterung der quantitativen Methodenausbildung.

\section{Herausforderungen}

\subsection{Neue Daten}

Die Datengrundlage für Analysen in der Politikwissenschaft hat sich in den letzten Jahren erheblich erweitert. In der klassischen quantitativen Forschung und Lehre werden hauptsächlich Daten aus der Umfrageforschung oder den amtlichen Statistiken verwendet. Mit dem Bedeutungsgewinn des Internets und sozialer Medien gibt es umfangreiche neue Datenquellen, welche breit digital verfügbar sind. In der sozialwissenschaftlichen Forschung und Lehre steigt hierdurch die Bedeutung neuer Datenquellen: umfangreiche Textdaten, digitale Spurendaten und Internetseiten (Blätte et al. 2018; Salganik 2018). In neueren Studien werden zum Beispiel Audiodaten automatisch ausgewertet (Dietrich et al. 2019) und weitere Formate wie Videoanalysen werden folgen. Die breiten Veränderungen, die sich aus neuen digitalen Daten und einfacher zugänglichen Analyseverfahren ergeben, werden allgemein unter den populären Begriffen Digitalisierung, Big Data oder Data Science diskutiert (Schnapp und Blätte 2018). Mit der zunehmenden Anzahl von Forschung im Bereich der Kommunikation, Deliberation und des Verhaltens von Akteuren im Internet geht eine Dezentralisierung der Datengenerierung einher. Diese Veränderungen haben direkte Implikationen für die Gestaltung der Lehre in politikwissenschaftlichen Studiengängen. Klassische Lehrformate quantitativer Methoden sollten überdacht und erweitert werden.

Die Erhebung klassischer Datenstrukturen, wie zum Beispiel Umfragedaten oder amtlicher Statistiken, ist aufwendig und ressourcenintensiv. Durch rechnergestützte Verfahren können mit neuen Techniken, ohne großen finanziellen oder zeitlichen Aufwand, Daten aus dem Internet generiert werden. Diese neuen Datenquellen und spezielle Analyseverfahren werden zum Beispiel angewendet bei der Analyse von Kommunikation in sozialen Medien (Barberá 2015) oder der Untersuchung von politischen Reden (Proksch und Slapin 2014). Die Datenquellen in Kombination mit

\footnotetext{
1 Wir gehen in diesem Beitrag auch an einigen ausgewählten Stellen auf die Entwicklungen und Erfahrungen aus dem Sommersemester 2020 ein, welches von Coronakontaktbeschränkungen geprägt war und somit als reines Onlinesemester durchgeführt wurde. In unserem Aufsatz plädieren wir für eine verstärkte Verwendung von E-Lernmaterialien, argumentieren aber aus einer Sicht von hybriden Modellen die Online- und Präsenzlehre verbinden. Der ursprüngliche Text ist bereits vor der COVID-Pandemie entstanden und hat nach unserer Überzeugung weiterhin Relevanz.
} 
automatisierten Analyseverfahren tragen zu einer Dezentralisierung von Datenanalyse in der Politikwissenschaft bei. Kleine Forschungsgruppen und auch Studierende können mit wenigen Ressourcen auf umfangreiche Datenmengen zugreifen und diese analysieren. Herausforderung von Methodenlehre im Zeitalter der Digitalisierung ist es daher, bei Studierenden die Fähigkeit zur Analyse dieser neuen Datenquellen zu entwickeln.

\subsection{Drei Entwicklungen}

In der politikwissenschaftlichen Lehre finden sich die Veränderungen durch Digitalisierung und Datenwissenschaft in drei Dimensionen wieder. Zunächst ist bei Studierenden ein hohes Interesse an der Verwendung der neuen Daten vorhanden. Hier verbinden sich schnell das substanzielle Interesse der Studierenden an politischen Prozessen und die Hoffnung, die breiten digitalen Datenquellen für eigene Arbeiten nutzen zu können. Gerade die neuen Möglichkeiten der Visualisierung großer Datenmengen wecken hier frühzeitig das Interesse der Studierenden.

Zweitens besteht die zunehmende Verantwortung der politikwissenschaftlichen Lehre darin, Studierende für die Verwendung dieser neuen Daten systematisch zu qualifizieren. Dynamiken der Digitalisierung betreffen Datenquellen in klassischen Arbeitsfeldern der Sozialwissenschaften: Verwaltung, Unternehmen und Forschung. Absolvent*innen sozialwissenschaftlicher Studiengänge sind zunehmend gefordert Analysen selbstständig umzusetzen. Der Umgang mit und die Analyse von neuen Datenquellen sind somit nicht mehr nur eine Spezialisierung von Studierenden, die sich auf quantitative Verfahren konzentrieren. Sie sind ein grundlegendes Element sozialwissenschaftlicher Hochschulbildung, welches in verschiedenen Abstufungen vermittelt werden muss. Alle Studierenden benötigen grundlegende Datenkompetenz für digitale Informationen. Zudem sollten sie die Möglichkeit erhalten, vertiefte Kenntnisse in ausgewählten Anwendungen, fortgeschrittenen statistischen Verfahren oder Programmierfähigkeiten erlernen zu können. Seit der Bologna-Reform liegt ein großer Schwerpunkt der Studienausrichtung auf der Beschäftigungsfähigkeit (Employability). In Verwaltung, NGOs, Beratungsfirmen und Forschung ist Datenkompetenz ein wichtiges Kriterium zur Einstellung. Absolvent*innen der Sozialwissenschaften sollen eigenständig Datenanalysen umsetzen und die Ergebnisse sprachlich breit zugänglich darstellen können. Sie können eine wichtige Brückenfunktion einnehmen zwischen Expertise ohne technische Ausbildung (z.B. Jurist*innen) und sehr technischer Expertise (Ingenieur*innen und Informatiker*innen).

Drittens gibt es eine breite Veränderung und Erweiterung des didaktischen Potenzials gerade im Bereich der Datenwissenschaft. Die schnellen Veränderungen in der Datenwissenschaft und das breite globale Interesse haben zu einem umfangreichen Angebot an digitalen Lernmaterialien geführt. Hier gibt es viele Internetkurse, Lernplattformen, Videotutorien, Materialien in sozialen Medien und Ähnliches. Lernen ist in diesem Feld ein kontinuierlicher und digital eingebetteter Prozess, in dem die Analysefähigkeiten an neuere Entwicklungen angepasst werden. Die vorliegenden Lernmaterialien lassen sich hervorragend in der universitären Lehre einsetzen und ermöglichen es, traditionelle Lehrformate zu erweitern. E-Lernen ist in diesem Bereich weit fortgeschritten, die E-Lernmaterialien sind konsolidiert und werden konti- 
nuierlich erweitert. Es bieten sich also hervorragende Möglichkeiten, die klassische hochschuldidaktische Trias aus Vorlesung, Seminar und Tutorium zu erweitern.

Die Herausforderung ist es, diese Veränderungen in die klassische Verbindung von Forschung und Lehre zu integrieren. Wie kann das gelingen? Wie vermitteln wir fachtheoretische Grundlagen, Forschungsdesign und Fähigkeiten zur Datenanalyse im digitalen Zeitalter? Was ist die beste Mischung aus Präsenzlehre, Projekten und digitalen Angeboten? Wie strukturieren wir ein Studium der Politikwissenschaft und die quantitative Methodenausbildung im digitalen Zeitalter so, dass Studierende am Ende des Studiums Fähigkeiten zum eigenen empirischen Arbeiten erworben haben?

\section{Methodenausbildung heute}

\subsection{Status quo}

Vor 15 Jahren wurde die Methodenausbildung in der Politikwissenschaft pointiert als „Stiefkind“ (Schnapp et al. 2004) bezeichnet und ein Mangel an Struktur diagnostiziert. Seither hat die Methodenlehre mit den Umstrukturierungen des BolognaProzesses einen großen Raum in der sozialwissenschaftlichen Ausbildung erhalten und ist fest im Curriculum sozialwissenschaftlicher Studiengänge verankert.

Unpassend ist der Begriff des Stiefkindes allerdings auch heute nicht, 15 Jahre nach der Mangeldiagnose. Methodenveranstaltungen sind oft isoliert und bei Studierenden nicht populär. Die Integration und Anwendung der Methodenkompetenz in der Breite des Curriculums ist häufig wenig systematisch und variiert sehr stark zwischen den Hochschulen. Einige Standorte haben den Methodenfokus umfangreich weiterentwickelt und zum Beispiel neue Stellen zur Methodenausbildung in der Politikwissenschaft eingerichtet. An anderen Standorten wird die Methodenausbildung mit breiten sozialwissenschaftlichen Einführungen für viele Studiengänge abgedeckt. Bei den Absolvent*innen der Politikwissenschaft in Deutschland variiert heute die tatsächliche Fähigkeit zur Anwendung von Methoden und die Sicherheit im Umgang mit den relevanten Programmen enorm zwischen den Standorten und Absolvent*innen. Der Anteil wissenschaftlicher Publikationen zur Konzeption und Durchführung von Methodenveranstaltungen bleibt überschaubar (Dreiack et al. 2016; Engel 2002; Kittel 2009).

Die Studieneingangsphase verpasst es oft, bei zunächst hauptsächlich fachlichinhaltlich interessierten Studierenden der Politikwissenschaft auch ein Interesse und Bewusstsein für methodische Arbeitstechniken zu wecken. Eine zentrale Aufgabe der Methoden- und Statistikausbildung in den ersten Studienjahren ist daher die Förderung von Motivation und Interesse. Die Methodenausbildung muss das Interesse der Studierenden wecken und ihnen direkte Bezüge zu eigenen politikwissenschaftlichen Anwendungen ermöglichen. Das Ziel ist weiterhin, „das negative Image der Methoden“ (Schnapp et al. 2004) langfristig abzubauen.

Wir sind davon überzeugt, dass die nachhaltige Fähigkeit zur Anwendung sozialwissenschaftlicher Methoden am besten durch eigenständige empirische Forschungsvorhaben vermittelt werden kann. Projektseminare können dafür einen hervorragenden Rahmen anbieten. Die Vermittlung von Grundlagen sozialwissenschaftlicher 
Methoden ist inzwischen an vielen deutschen Hochschulen gut etabliert. Es mangelt an Motivation, der Einbindung von Methoden in inhaltlichen Veranstaltungen und der Öffnung für neue Daten.

\subsection{Klassische Lernmaterialien, Lehrinhalte und neue Herausforderungen}

Welche Herausforderungen stellen sich an die Konzeption und Umsetzung von Methodenlehre durch neue Daten und Datenwissenschaft? In etablierten Lehrformaten werden allgemeine sozialwissenschaftlichen Einführungen in statistische Verfahren (z. B. Agresti und Finlay 2014; Diez et al. 2019) am Beginn des Studiums verwendet und Bücher der Ökonometrie (z. B. Wooldridge 2015) in der vertiefenden Ausbildung im Masterstudium genutzt. In der sozialwissenschaftlichen Methodenausbildung gibt es einen Grundkanon wichtiger Analyseverfahren und diese konzentrieren sich auf ein überschaubares $\mathrm{Ma} ß$ an deskriptiven und multivariaten statistischen Verfahren, welche vertiefend behandelt werden. Unterschiede zwischen Standorten in Deutschland existieren bei der fortgeschrittenen Ausbildung im Bachelor und Master. Einige Standorte haben eine starke Profilbildung im Bereich quantitativer Methoden entwickelt und bieten bereits spezialisierte Masterprogramme in Datenwissenschaft an. An anderen Standorten ist das Methodenspektrum pluraler und eine vertiefte Ausbildung wird über externe Kurse von GESIS Training, der ECPR Methods School oder anderen Anbietern abgedeckt.

Im Bereich der Datenwissenschaft sind Datenquellen und Analyseverfahren umfangreicher und heterogener. Datenquellen umfassen auch Textdaten, Bilder, Audio und Video. Zur Analyse wird ein breites Spektrum von Verfahren des maschinellen Lernens genutzt. Die Anwendung verlangt die Nutzung einer modernen Programmiersprache wie R oder Python. Dies führt zu neuen Herausforderungen in der Konzeption von Lehrveranstaltungen. Etablierte Lehrinhalte müssen Platz für neue Verfahren einräumen. Neuere Lehrbücher verdeutlichen diese Veränderung. So gibt das Lehrbuch von Imai (2018) bereits frühzeitig einen Überblick zu neueren Verfahren (Analysen von Netzwerken, Texten und räumlichen Daten), bevor es die Grundlagen der statistischen Inferenz vermittelt. Zudem wird in dem Lehrbuch ein Analyseprogramm (R) konsequent bei der Vermittlung der Verfahren verwendet.

Es gibt immer weniger einen Kanon der wichtigsten Verfahren, wie es lange die vertiefte Ausbildung zur Regressionsanalyse war. Lehre in der Politikwissenschaft in Deutschland und die Standorte stehen vor der Herausforderung, den Methodenmix in Studienprogrammen neu zu definieren. Welche Daten und Verfahren sind am Standort besonders relevant? Wo liegen die eigenen Kompetenzen eines Instituts und der Universität? Aus unserer Sicht sollten Standorte noch stärker das eigene Methodenprofil schärfen und konsequent in der Lehre abbilden. Eine weiter zunehmende Heterogenität der Ausbildung in fortgeschrittenen Methoden in Deutschland ist eine der Folgen. Neben dieser Herausforderung einer Profilbildung in der Methodenausbildung verbleibt die Schwierigkeit, Studierende der Sozialwissenschaft, welche zunächst substanziell interessiert sind, für die systematische Anwendung von Methoden zu motivieren. 


\subsection{Herausforderung Motivation}

Bei Studierenden Interesse für sozialwissenschaftliche Methoden zu wecken, ist eine Kernaufgabe in der Studieneingangsphase. Die Gründe hierfür sind simpel: Je mehr Bewusstsein für und Interesse an politikwissenschaftlichen Methoden Studierende entwickeln, desto größer ist die Aufmerksamkeit, die sie diesen entgegenbringen. Hierdurch ergeben sich Synergien für das politikwissenschaftliche Studium und die Ziele der sozialwissenschaftlichen Ausbildung. Bei starkem Interesse an Methoden, der Fähigkeit zum Selbststudium und der Umsetzung von Methoden in den ersten Semestern des Studiums steigt die Qualität des wissenschaftlichen Arbeitens in weiteren Studienabschnitten: bei der Seminarbeteiligung und bei Haus- bzw. Abschlussarbeiten. Wenn Studierende bereits am Beginn Fähigkeiten zum methodischen Arbeiten und eigenständigen Lernen entwickeln, kommt dies allen Lernenden und Lehrenden zugute.

Elementare Aspekte der Motivation von Studierenden für die Methodenlehre fassen Lewthwaite und Nind (2016) in einer Studie zusammen. Aus Expert*inneninterviews haben sich für die Autorinnen drei Ansätze herauskristallisiert, welche zu besseren Lern- und Lehrerfahrungen führen. Der erste Schritt ist, Forschung sichtbar zu machen. Dies bedeutet, dass Forschungsideen und Daten verknüpft werden im Zusammenhang mit methodischem Arbeiten. Den Studierenden soll der gesamte Forschungsprozess präsentiert werden und ein problembasiertes Lernen stattfinden. Hierfür kann bereits in den ersten Sitzungen mit der Datenarbeit begonnen werden, z. B. in Verknüpfung mit Analyseprogrammen.

Das zweite Thema umfasst das Lernen durch eigene Forschung. Hierbei sind Daten ein zentrales Element. Es geht nicht nur um die Analyse, sondern auch um die Generierung und die Aufbereitung von Daten. Die Expert*innen betonen die Wichtigkeit von realen Daten, welche aus relevanten Forschungsvorhaben ausgewählt wurden. Im Idealfall können die Studierenden Daten selbst generieren, um ein gutes Lernergebnis zu erzielen. Durch die Generierung eigener Daten entsteht für die Studierenden das Gefühl von Eigenverantwortung und Eigentümerschaft. Dies steigert die Motivation beträchtlich, anstatt lediglich Forschung anderer nachzuvollziehen. Hier können durch neue Datenstrukturen viele Potenziale freigesetzt werden.

Das dritte Thema umfasst die Reflexion von Forschungspraktiken. Durch die beschriebene Anwendung sollen Studierende die Fähigkeit erwerben, Forschungsprojekte zu reflektieren, besser zu verstehen und kritisch zu hinterfragen. Hier decken sich die Ansicht der Expert*innen und unsere Perspektive. Ein Verstehen, die Fähigkeit, zu reflektieren und zu hinterfragen, kann nur mit der Durchführung eigener Forschungsprojekte erlangt werden. Wenn diese drei Paradigmen in den Lernprozess integriert werden, können quantitative Methoden nicht mehr losgelöst von der Theorie behandelt werden (Kittel 2009). Um die Lehre in konkreten Forschungsprojekten zu gestalten, muss es sich daher um übergeordnete politikwissenschaftliche Themen handeln (Dreiack et al. 2016). Unseres Erachtens können die Herausforderungen der Motivation sehr gut durch die Implementierung neuer Datenquellen in der Methodenlehre bewältigt werden. Hierfür müssen aber neue Schwerpunkte in der Lehre gesetzt werden. 


\section{Neue Schwerpunkte}

Technisch bieten die neuen Datenquellen und neue Analyseprogramme viele Potenziale für die Anpassung der Methodenausbildung. Es kann eine noch breitere Anbindung an Forschungsfragen und Interessen der Studierenden erfolgen. Didaktisch sind es ein Fokus auf projektorientierte Lehre, aktives Lernen und die systematische Einbindung von E-Lernmaterialien, welche unserer Meinung nach eine zeitgemäße Aktualisierung der Methodenausbildung ermöglichen und das Interesse der Studierenden erhöhen.

\subsection{Neue Daten}

Durch die Integration von neuen Datenquellen in das Curriculum der Methodenlehre ergeben sich zwei Vorteile: Zum einen spiegelt die Lehre die Veränderungen der Disziplin wider. Klassische Datengrundlagen wie Umfragen und amtliche Statistiken werden zwar weiter in der Politikwissenschaft verwendet. Allerdings ist eine starke Fokussierung auf diese klassischen Daten in der quantitativen Methodenlehre bei der Breite neuer digitaler Daten nicht mehr zeitgemäß (Zwick 2016). Die stetig wachsende Bedeutung von Big Data und alternativen Datenquellen muss auch in der Lehre berücksichtigt werden. Hierdurch werden Studierende umfassender auf die Anforderungen eines breiten Arbeitsmarktes vorbereitet und Studiengänge können den Anspruch nach Employability der Absolvent*innen besser erfüllen. Die Aufnahme dieser Datenquellen ermöglicht zudem eine einfachere umfassende Umsetzung von Forschungsprojekten durch Studierende in Lehrveranstaltungen: von der Datenerhebung bis zur Analyse.

Durch die Dezentralisierung der Datengenerierung und mit Big Data ergeben sich vielfältige Potenziale für die Methodenlehre. Die Verwendung von neuen Datenquellen, welche durch Studierende selbst aufbereitet werden können, wirkt motivierend. Gerade die Daten aus sozialen Medien haben einen direkten Bezug zur Lebenswelt der digitalen Generation (Digital Natives). Mit geeigneten Lehrmethoden sind Studierende bereits nach wenigen Lerneinheiten in der Lage, eigenständig reale Daten für Forschungsprojekte zu erheben. Dies kann dezentral und ohne großen Ressourcenaufwand umgesetzt werden. Zudem erweisen sich Themenbereiche der sozialen Medien an der Schnittstelle zwischen Politik- und Kommunikationswissenschaft als attraktiv und ermöglichen es, auch Studierende an quantitative Methoden heranzuführen, welche klassische Seminare oder Vorlesungen mit statistischer Ausrichtung eher nicht belegen würden. Die aktuelle Breite an digitalen Datenquellen, neueren Analyseverfahren, Auswertungsprogrammen und E-Lernmaterialien ermöglicht es, didaktische Konzepte und Lernperspektiven zu erweitern.

\subsection{Programmieren}

Neue Daten verlangen eine Neujustierung bei der Vermittlung von Fähigkeiten zur Anwendung von Analyseprogrammen. Klassisch war dieser Bereich von Tabellenkalkulation (Excel oder LibreOffice) und der Vermittlung eines Statistikprogrammes (SPSS oder Stata) bestimmt. Im Bereich neuer Daten haben diese Statistikprogram- 
me kaum Bedeutung. Hier werden heute Sprachen wie R oder Python verwendet. Neue Verfahren des maschinellen Lernens werden vorrangig in diesen Sprachen implementiert und in verschiedenen Wissenschaftsdisziplinen verwendet. Die Sprachen ermöglichen den einfachen Zugang zu neuen Daten in Erweiterungen (Paketen). Sie sind zudem kostenlos verfügbar (Open Source) und es existiert ein umfangreiches digitales Angebot von freien und kommerziellen Tutorien, Hilfeseiten sowie Internetkursen. Damit sind die Sprachen auch nach dem Studium einfach nutzbar und bieten einen breiten Rahmen für die berufliche Weiterbildung. Wir sind daher überzeugt, dass das Vermitteln und Erlernen moderner Sprachen wie R oder Python sehr sinnvoll für die Ermöglichung des Zugangs zu digitalen Daten ist (Munzert 2018).

Die Methodenausbildung muss sich also dahin verändern, dass für die Vermittlung von grundlegenden Fähigkeiten im Programmieren mehr Zeit aufgebracht wird. Der Fokus sollte dabei ganz klar auf Datenanalyse, Visualisierung und sozialwissenschaftlichen Anwendungen liegen. Ein grundlegendes Verständnis über Datenstrukturen und von einfachen Programmierkonzepten ist notwendig für die Aufbereitung und Analyse neuer digitaler Daten. Diese Veränderungen betreffen viele Studiengänge, sodass sich auf Fakultäts- oder Universitätsebene die Bündelung von Kompetenzen und Lehrangeboten in einem Zentrum für Datenwissenschaft anbietet.

Als Fachdisziplin kann die Politikwissenschaft durch die Nutzung von OpenSource-Sprachen zum Ziel der offenen Wissenschaft (Open Science) beitragen. Diese Bewegung ist bestrebt wissenschaftliche Erkenntnisse und Elemente des Forschungsprozesses wie Daten und Skripte frei verfügbar sowie breit zugänglich zu machen. Die Politikwissenschaft kann gerade im Bereich der quantitativen Datenanalyse noch stärker zu einer offenen Wissenschaft beitragen, indem gut aufbereitete und umfangreich dokumentierte Replikations- und Lehrmaterialien digital veröffentlicht werden. Studierenden wird so außerdem ein einfacherer Zugang zum wissenschaftlichen Arbeiten und zu Elementen des Forschungsprozesses ermöglicht.

\subsection{Projektorientierte Lehre}

Als ein zentrales didaktisches Element der Methodenlehre schlagen wir die Konzentration auf Projektseminare vor. Durch die Kombination von inhaltlichen Schwerpunkten, digitalen Materialien und Methoden soll bei Studierenden Interesse am quantitativen wissenschaftlichen Arbeiten geweckt werden. Die Verknüpfung von Theorie und Methodik ermöglicht zudem eine fundierte Auseinandersetzung mit beiden Schwerpunkten. Ziel hierbei ist es, dass am Ende des Seminars die Studierenden sich mit den theoretischen Annahmen, bisherigen Erkenntnissen und Perspektiven eines Themas auseinandergesetzt haben. Dabei sollen Studierende methodisches Vorgehen hinterfragen können und eine eigene Studie im Themengebiet umsetzen können. Digitale Materialien ermöglichen Phasen des selbstständigen Lernens während des Projektseminares.

Neu an der Konzeption dieser Seminare ist nicht der Projektcharakter per se, sondern vielmehr die Reihenfolge der behandelten Themen und Schwerpunkte. Das statistische Wissen sowie Programmierkenntnisse werden nicht mehr skizzenhaft und losgelöst von der thematischen Materie vermittelt, sondern stringent an politikwissenschaftlichen Themen illustriert und angewendet. Die Relevanz der Methoden 
wird hierdurch deutlicher im Vergleich zu Veranstaltungen, welche primär auf die Vermittlung von methodischem Wissen fokussiert sind und ausgewählte Anwendungsbeispiele verwenden.

Der Aufbau des Seminars und die Lernkurve sind mit Pournas' (2017) Seminarstruktur nach Bloom verwandt. Unsere Perspektive unterscheidet sich jedoch durch den stringenten theoretischen Fokus. Anders als Munzert (2014) nehmen wir hier eine Komplexionsreduktion bei Programmierfähigkeiten in Kauf. Ziel eines Projektseminars ist es nicht, den Studierenden umfassende R-Fähigkeiten zu vermitteln, sondern durch die Einbindung in den Forschungsprozess die Motivation und das Interesse zu schüren. Durch die Kombination von fachlichen Fragen mit E-Lernmaterialien werden die Studierenden in die Lage versetzt, wichtige Fähigkeiten eigenständig zu erwerben.

In Abb. 1 stellen wir den Ablauf eines Projektseminares dar, welches einen fachlichen Fokus und die Vermittlung von Methodenkenntnissen kombiniert. Der Seminarplan unterteilt sich in vier Blöcke. Im ersten Block werden die theoretischen bzw. thematischen Grundlagen gelegt. Die Sitzungen sind „klassisch“ strukturiert mit der Lektüre von Texten in der Vorbereitung sowie Diskussionen im Seminar. Erste grundlegende Softwareschritte werden durch E-Lerneinheiten im Selbststudium absolviert. Der zweite Block dient der methodischen Kompetenzvermittlung sowie dem Erwerb von Softwarefähigkeiten. Hier bietet sich eine Kombination aus Selbstlernmodulen und optionalen Präsenzzeiten an (z. B. offene Werkstätten). In den Selbstlernmodulen (E-Lernkurse zu Programmierfähigkeiten und Analyseverfahren) werden die speziellen Fähigkeiten von den Studierenden selbstständig erworben und

Block 1: Theoretische Grundlagen / Einführung in $\mathbf{R}$

Dauer: 4 Sitzungen

Ziel: Diskussion zentraler Theorien und Erkenntnisse im Forschungsfeld \& Einführung in R

Beschreibung: Lektüre von Texten / Studien sowie kleinere R-Tutorials im Selbststudium

Block 2: Vertiefung R / direkte Anwendung

Dauer: 5 Sitzungen

Ziel: Zentrale R-Fähigkeiten im Bereich des Datensatzmanagements / Visualisierung und Analyse.

Beschreibung: Erlernen der Fähigkeiten im Selbststudium zuhause. Direkte Anwendung im Seminar auf Datensätze passend zur thematischen Ausrichtung

Block 3: Ausarbeitung der Forschungsprojekte

Dauer: 3 Sitzungen

Ziel: Studierende erarbeiten ihre Forschungsprojekte und setzen diese um.

Beschreibung: Keine Präsenzzeit, Möglichkeit der offenen Werkstätten oder Sprechstundenangebote

Konferenz-Simulation

Präsentation von Studierenden(-teams) à 15min, Kommentierung der Papiere/Präsentationen durch andere Studierende (Teams), Diskussion

(

$\underbrace{2}$

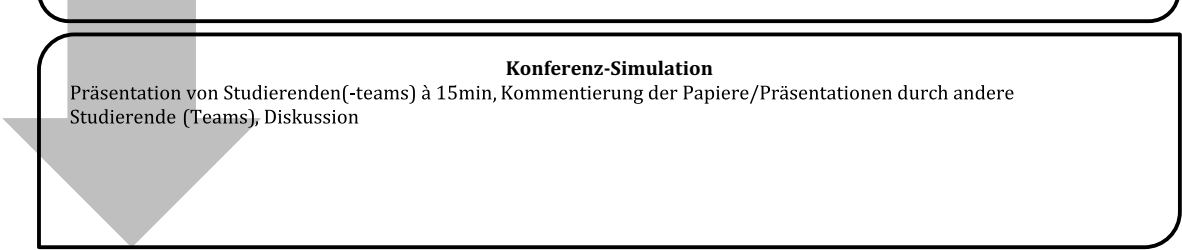

Abb. 1 Struktur Projektseminar mit Methodenbezug 
vertieft. Im Anschluss können diese direkt für die eigene Forschungsfrage angewendet werden. Lehrende sind Lotsen bei der Auswahl der Selbstlernmodule und schaffen einen Austausch in Präsenzveranstaltungen oder digitalen Sitzungen, der das eigene Arbeiten der Studierenden strukturiert. Der dritte Block dient zur Fertigstellung der Forschungsvorhaben sowie der Präsentationsvorbereitung. Abschließend werden im vierten Block (Anzahl der Sitzungen nach Seminargröße) die Ergebnisse im Panelformat vorgestellt. Rückmeldungen können dann in der Hausarbeit aufgegriffen werden.

Je nach zeitlicher Gestaltung, kann der gesamte Durchlauf des Forschungszyklus (von Fragestellung bis hin zur Beantwortung in einer Hausarbeit) für die Studierenden zu herausfordernd sein. Hier bieten sich alternative Prüfungsformate an. Eine Möglichkeit ist es, die Studierenden Replikationen von bestehenden Studien erarbeiten zu lassen. Nachteile dieses Vorgehens liegen in der mangelnden eigenen Verwirklichung oder Kreativität, Vorteile darin, dass Wissenschaft etwas ,entzaubert“ wird und nahbarer bzw. verständlicher wird. Eine weitere Möglichkeit ist es, Studierende aufzufordern, bereits geschriebene Hausarbeiten mit einem quantitativen Schwerpunkt neu aufzulegen.

Der Vorteil von Projektorientierung in der Lehre ist eine direkte Umsetzung des Erlernten. Besonders die zeitliche Abfolge erhöht den Lernerfolg. Es wird nicht erst eine große Menge Wissen akkumuliert, um dieses am Ende des Semesters in der Prüfungsleistung anzuwenden. Das Gelernte wird zeitnah angewendet. Die Verantwortung für ein eigenständiges Forschungsprojekt fördert das Interesse der Studierenden über die Dauer des Semesters. Dieses Vorgehen schafft bei den Studierenden das Vertrauen, eigene Forschung zu produzieren und Methoden unmittelbar anzuwenden. Ein motivierender Faktor ist dies gerade im Bachelorstudium, in welchem noch immer vieles nur reproduziert wird.

Diese Seminarkonzeption ist stark an die Ideen des Flipped Classroom angelehnt. Auch hier wird ein Großteil der Wissensakkumulation im Selbststudium erbracht und die Anwendung in den Seminar- oder Vorlesungskontext verlegt. Der Einsatz in den Sozialwissenschaften hat sich hier als vielversprechend erwiesen, gerade in den Methodenveranstaltungen. Vor allem im Hinblick auf Anwendungsfragen wurden bessere Lernergebnisse bei Studierenden erzielt (Goerres et al. 2015). Beim Konzept der Projektseminare handelt es sich nicht um einen reinen Flipped Classroom. Die Motivation der Studierenden für methodische und statistische Themen erfolgt nicht allein über die didaktische Methode, sondern durch die Koppelung mit der Thematik. Ähnlich wie beim Flipped Classroom werden stärker digitale Lernmaterialien zur Vorbereitung und Begleitung der Präsenzveranstaltungen genutzt.

\subsection{Selbstlernmodule}

Im Bereich der Datenwissenschaft hat sich in den letzten fünf Jahren ein umfangreiches Angebot an digitalen Lernmaterialien entwickelt. Dies ermöglicht es, Projektseminare durch Selbstlernmodule zu ergänzen. Das Angebot umfasst frei zugängliche Lernmaterialien wie OpenIntro Statistics oder Data Carpentry und kommerzielle Anbieter im Bereich der Masseninternetkurse wie SAGE Campus, DataCamp oder Johns Hopkins Data Science bei Coursera. Einige der Anbieter richten sich direkt 
an Universitäten, welche diese Angebote des E-Lernens in das bestehende Lehrangebot integrieren können. Diese Angebote stellen umfangreiches Material sowohl für beginnende als auch fortgeschrittene Lernende bereit. Die Materialien umfassen sowohl einfache erste Schritte der Datenaufbereitung und grundlegende Programmierkompetenz im Bereich der Datenwissenschaft als auch spezialisierte Module zu vertiefenden Anwendungsverfahren. Diese Materialien können fachliche und methodische Veranstaltungen sehr gut ergänzen. Da diese sich an ein sehr breites $\mathrm{Pu}-$ blikum richten, ist es wichtig, dass Lehrende der Politikwissenschaft Bezüge und Anwendungen der Fachdisziplin in eigenen Lernelementen und Anwendungsbeispielen verdeutlichen.

In Tab. 1 geben wir Beispiele zu prominenten Anbietern von E-Lernmaterialien und veranschaulichen das Angebot für Lehrende. Die Tabelle zeigt, dass es verschiedene didaktische und technische Ansätze gibt. Einige Angebote wie OpenIntro Statistics und $\mathrm{R}$ for Data Science orientieren sich am klassischen Lernformat des Lehrbuches. In einem Text werden Verfahren der Datenanalyse vorgestellt und in Codebeispielen Anwendungen veranschaulicht. Andere Anbieter wie DataCamp konzentrieren sich stark auf eine interaktive Wissensvermittlung im Web. Dabei werden kurze Videotutorien und interaktive Übungen im Web kombiniert. Lernende sehen zunächst eine kurze Videoeinführung zu einem Verfahren und wenden dieses sofort in Beispielen an. Die Entwicklung des Angebots an E-Lernmaterialien ist sehr dynamisch, sodass wir hier nur eine temporäre Bestandsaufnahme machen können. Sicher ist allerdings, dass dieses Angebot weiter stark wächst und sich hoffentlich konsolidiert.

Mit den umfangreichen digitalen Materialien im Internet besteht eine Herausforderung der Lehre an Universitäten heute gerade darin, die Potenziale des E-Lernens mit dem Präsenzcharakter universitärer Lehre zu verbinden. Die Erfahrungen des Sommersemesters 2020 haben gezeigt, dass, wenn es keine Alternative gibt, diese Herausforderung auch gemeistert werden kann. Wenn auch durch spontane Kraftakte der Lehrenden wurde das „Online-Corona-Semester“ zu großen Teilen von allen Beteiligten einigermaßen erfolgreich über die Bühne gebracht. Die Methodenausbildung umfasst traditionell einen hohen Anteil frontaler Lehre. Viele dieser grundlegenden Lerneinheiten lassen sich durch E-Lernen abdecken. Dabei ist es besonders die Kombination aus freien und kommerziellen E-Lerneinheiten mit eigenem Material, welche den Reiz der neuen Angebote darstellt. Die passende Mischung aus kommerziellen und freien Materialien des E-Lernens zu finden, ist die Herausforderung aller Hochschulen. Diese Mischung wird sich zwischen Standorten unterscheiden und hängt ab von finanziellen Ressourcen, kulturellen Präferenzen sowie der Bereitschaft, eigene E-Materialien zu entwickeln. Dank der Erfahrungen und Entwicklungen aus dem erzwungenen digitalen Sommersemester 2020 ist zu erwarten, dass für einen nachhaltigen Mix aus Präsenz- sowie digitaler Lehre die Akzeptanz bei Lehrenden, Studierenden sowie Verwaltung gestiegen ist.

Wir als Fachdisziplin müssen erarbeiten, wo diese breiten Angebote spezielle Aspekte der Politikwissenschaft nicht abdecken können und wo wir eigene offene Lernmaterialien entwickeln sollten. Freie und kommerzielle Angebote des E-Lernens richten sich zumeist an ein sehr breites Publikum. Diese decken nicht spezielle Fragestellungen in den Teilgebieten der Politikwissenschaft ab. Hier ist das Fach 
Tab. 1 Beispiele Anbieter E-Lernmaterialien

\begin{tabular}{|c|c|c|c|c|c|}
\hline Anbieter & Fokus & Format & Aufwand & Vorteile & Nachteile \\
\hline $\begin{array}{l}\text { R for Da- } \\
\text { ta Science } \\
\text { (Wickham } \\
\text { und Grole- } \\
\text { mund 2016) } \\
\text { (Kostenlos/ } \\
\text { Englisch) }\end{array}$ & $\begin{array}{l}\mathrm{R} \\
\text { Datenanalyse } \\
\text { Visualisierungen } \\
\text { Statistische } \\
\text { Verfahren }\end{array}$ & $\begin{array}{l}\text { Buch und } \\
\text { freie Web- } \\
\text { seite (r4ds. } \\
\text { had.co.nz) }\end{array}$ & $\begin{array}{l}\text { Gering bis } \\
\text { moderat } \\
\text { Implementierung } \\
\text { im Seminar- } \\
\text { kontext Bezug } \\
\text { SoWi }\end{array}$ & $\begin{array}{l}\text { Individuelles } \\
\text { Lerntempo } \\
\text { Große Aus- } \\
\text { wahl Alterna- } \\
\text { tiven }\end{array}$ & $\begin{array}{l}\text { Statisch } \\
\text { Andere An- } \\
\text { gebote nur } \\
\text { kommerziell } \\
\text { oder Biblio- } \\
\text { theken }\end{array}$ \\
\hline $\begin{array}{l}\text { DataCamp } \\
\text { (Kostenlos } \\
\text { für Semi- } \\
\text { nargruppen/ } \\
\text { Englisch) }\end{array}$ & $\begin{array}{l}\text { R und Python } \\
\text { Datenmanage- } \\
\text { ment } \\
\text { Visualisierungen } \\
\text { Statistische } \\
\text { Verfahren }\end{array}$ & $\begin{array}{l}\text { Kurze Vi- } \\
\text { deotutorien } \\
\text { Interaktive } \\
\text { R-Sitzung } \\
\text { Webseite } \\
\text { Kurzübungen }\end{array}$ & $\begin{array}{l}\text { Gering bis } \\
\text { hoch - abhän- } \\
\text { gig von eigener } \\
\text { Kursteilnahme }\end{array}$ & $\begin{array}{l}\text { Hohes didak- } \\
\text { tisches Niveau } \\
\text { und sehr gute } \\
\text { Dozierende } \\
\text { Aktuelle Ent- } \\
\text { wicklungen }\end{array}$ & $\begin{array}{l}\text { Kein Einfluss } \\
\text { auf Inhalte } \\
\text { Kein Nach- } \\
\text { schlagen } \\
\text { Kommerziell } \\
\text { (Kosten, } \\
\text { Abhängigkeit) }\end{array}$ \\
\hline $\begin{array}{l}\text { Eigene } \\
\text { Materialien } \\
\text { (Kostenlos/ } \\
\text { Sprache } \\
\text { nach Wahl) }\end{array}$ & $\begin{array}{l}\text { R } \\
\text { Eigener Fo- } \\
\text { kus wählbar }\end{array}$ & $\begin{array}{l}\text { Bsp. } \\
\text { R-Mark- } \\
\text { down }\end{array}$ & $\begin{array}{l}\text { Sehr hoch } \\
\text { (beim ersten } \\
\text { Mal) }\end{array}$ & $\begin{array}{l}\text { Individueller } \\
\text { Inhalt } \\
\text { Kombination } \\
\text { Theorie, } \\
\text { Methoden } \\
\text { und R } \\
\text { Rechte beim } \\
\text { Lehrenden }\end{array}$ & $\begin{array}{l}\text { Multimedia- } \\
\text { inhalte extrem } \\
\text { aufwendig }\end{array}$ \\
\hline $\begin{array}{l}\text { OpenIntro } \\
\text { Statistics } \\
\text { (Kostenlos/ } \\
\text { Englisch) }\end{array}$ & $\begin{array}{l}\text { Statistische } \\
\text { Verfahren mit } \\
\text { R-Anwen- } \\
\text { dung }\end{array}$ & $\begin{array}{l}\text { Lehrbuch } \\
\text { mit be- } \\
\text { gleitenden } \\
\text { Übungen }\end{array}$ & Gering & $\begin{array}{l}\text { Didaktisches } \\
\text { Konzept } \\
\text { Multimedia- } \\
\text { inhalte }\end{array}$ & $\begin{array}{l}\text { Fokus klassi- } \\
\text { sche statisti- } \\
\text { sche Verfah- } \\
\text { ren } \\
\text { Wenig Inter- } \\
\text { aktion }\end{array}$ \\
\hline $\begin{array}{l}\text { Sage Cam- } \\
\text { pus } \\
\text { (Englisch) }\end{array}$ & $\begin{array}{l}\text { R und Python } \\
\text { Aufbereitung } \\
\text { und Visua- } \\
\text { lisierung } \\
\text { Daten }\end{array}$ & Videotutorien & Gering & $\begin{array}{l}\text { Fokus Sozial- } \\
\text { wissenschaf- } \\
\text { ten }\end{array}$ & $\begin{array}{l}\text { Wenig klassi- } \\
\text { sche Verfah- } \\
\text { ren } \\
\text { Wenig Inter- } \\
\text { aktion } \\
\text { Kommerziell }\end{array}$ \\
\hline $\begin{array}{l}\text { Softwarel } \\
\text { Data Car- } \\
\text { pentry } \\
\text { (Kostenlos/ } \\
\text { Englisch) }\end{array}$ & $\begin{array}{l}\text { R und Python } \\
\text { „software } \\
\text { tools“ } \\
\text { Datenmanage- } \\
\text { ment }\end{array}$ & $\begin{array}{l}\text { Struktur } \\
\text { und Ma- } \\
\text { terial für } \\
\text { Kurzsemi- } \\
\text { nare }\end{array}$ & Moderat & $\begin{array}{l}\text { Lernenden- } \\
\text { zentriert }\end{array}$ & $\begin{array}{l}\text { Fokus auf } \\
\text { Naturwissen- } \\
\text { schaften }\end{array}$ \\
\hline $\begin{array}{l}\text { Swirl } \\
\text { (Kostenlos/ } \\
\text { Englisch) }\end{array}$ & $\begin{array}{l}\text { R-Paket } \\
\text { Vorgefertigte } \\
\text { Lerneinhei- } \\
\text { ten } \\
\text { Möglichkeit } \\
\text { zur eigenen } \\
\text { Programmie- } \\
\text { rung }\end{array}$ & $\begin{array}{l}\text { In R/ } \\
\text { RStudio } \\
\text { integrierte } \\
\text { Vorlagen } \\
\text { für Tutori- } \\
\text { en }\end{array}$ & $\begin{array}{l}\text { Gering bis sehr } \\
\text { hoch } \\
\text { Nutzung be- } \\
\text { stehender } \\
\text { Tutorien oder } \\
\text { eigene Tutorien } \\
\text { programmieren }\end{array}$ & $\begin{array}{l}\text { Implementiert } \\
\text { in RStudio } \\
\text { Hoher Ein- } \\
\text { fluss auf } \\
\text { Inhalte }\end{array}$ & $\begin{array}{l}\text { Nur textba- } \\
\text { siert } \\
\text { Keine Interak- } \\
\text { tion }\end{array}$ \\
\hline
\end{tabular}


in der Verantwortung, disziplinspezifische Lern- und Forschungsmaterialien breit zugänglich zu machen (Open Educational Resources und Open Science). Digitale Plattformen vereinfachen die Zusammenarbeit bei der Erstellung dieser Materialien und deren Veröffentlichung.

Gerade im Bereich der Datenwissenschaft bietet sich die Verwendung von Konzepten des aktiven Lernens und des Flipped Classroom an, welche die Vorzüge der neuen E-Lernmaterialien und von Präsenzveranstaltungen verknüpfen (Goerres et al. 2015). Lehrende wählen ein Portfolio von E-Lernmaterialien aus, welches die Studierenden zur Vor- und Nachbereitung von Sitzungen nutzen. Überblickswissen wird außerhalb der Präsenzveranstaltung in Videotutorien und interaktiven Lernplattformen erworben. Diese breiten Einführungen ergänzen Lehrende durch beispielhafte Anwendungen der Politikwissenschaft und kleine Beispiele der eigenen Forschung. Anwendungen und Beispiele sollen vollständig digital dokumentiert und replizierbar sein. Die Veranstaltungen dienen dann zum Vernetzen der Studierenden in Lerngruppen, zum Abgleichen der Lernfortschritte, zur Rückmeldung über die Zugänglichkeit der Konzepte, zur Visualisierung und Präsentation des Erlernten und Erarbeiteten. Unsere Erfahrung zeigt, dass es oftmals die kleinen Schritte am Beginn des Lernens sind, die regelmäßige Rückmeldung und Interaktion erfordern. Gemeinsames Lernen schafft einen sozialen Rahmen, in dem das Selbstlernen strukturiert und motiviert wird.

In eigenen Lehrveranstaltungen haben wir diese Konzepte des aktiven Lernens mit E-Lernmaterialien angewendet. Wir haben die vorgestellten Konzepte in Präsenzveranstaltungen erprobt sowie im Sommersemester 2020 unter den schwierigen Bedingungen der Coronakontaktbeschränkungen auch rein digital durchgeführt. Studierende arbeiten dabei, auch in der Präsenzzeit, am eigenen Rechner. Eine Seminarsitzung wird in mehrere kurze Blöcke geteilt, bei der sich kurze Überblicksdarstellungen durch die Lehrenden, das aktive Bearbeiten von Übungen am Rechner und das gemeinsame Diskutieren von Lösungswegen abwechseln. Die Lehrenden definieren den Rahmen, unterstützen die Lernenden dann direkt am Rechner und fassen Lösungsansätze sowie Fallstricke der Arbeit am Rechner zusammen. Die systematische Vorstellung der Inhalte erfolgt durch die E-Lernmaterialien bei der Vor- und Nachbereitung der Sitzung durch die Studierenden. Kleine Tricks wie die Verwendung von Klebezetteln zum Signalisieren von Unterstützungsbedarf und als Rückmeldung zwischen den Lerneinheiten fördern dabei das interaktive Lernen.

Reine Onlineseminare, wie sie völlig überraschend im Sommersemester 2020 durch die Coronakontaktbeschränkungen erforderlich wurden, bringen weitere Herausforderungen mit sich, welche losgelöst von methodischen oder statistischen Seminaren sind und perspektivisch im (politik-)wissenschaftlich didaktischen Diskurs aufgegriffen werden müssen. Die in unserem Beitrag immer wieder betonte Interaktion mit den Studierenden unterliegt anderen Hürden und ein reines E-Lernen, wie es im Coronasemester erforderlich war, verändert das Zusammenarbeiten zwischen Lehrenden und Studierenden erheblich. 


\section{Fazit}

Methodenlehre steht weiter vor der Herausforderung, gerade am Beginn des Studiums Motivation und Bewusstsein für die Verwendung sozialwissenschaftlicher Methoden zu vermitteln. Gleichzeitig ist die Bedeutung von Forschungsdesign, neuen Daten und modernen Analyseverfahren in den letzten zwei Jahrzehnten stark gestiegen. Diese Entwicklungen und Herausforderungen stellen wir in diesem Beitrag dar. Wir betonen besonders die Relevanz von didaktischen Konzepten des aktiven Lernens, Potenziale des E-Lernens und die Rolle von Projektseminaren. Hierdurch werden Studierende für diverse Realitäten auf dem Arbeitsmarkt nach dem Studium vorbereitet.

Aus unserer Sicht bieten gerade Projektseminare die Möglichkeit, den gesamten Forschungsprozess umzusetzen. Diese Seminare werden mit Angeboten des E-Lernens begleitet. Studierende vertiefen damit die notwendige Methodenkompetenz in Selbstlerneinheiten zur Umsetzung ihres Projektes. Am Ende eines solchen Projektseminares sind die Studierenden idealerweise in der Lage, eigene Daten aus dem Internet zu generieren und diese auf eine politikwissenschaftliche Fragestellung anzuwenden.

Für die Lehrenden stellen sich zwei Herausforderungen. Zum einen sind sie noch stärker gefordert die Erkenntnisse der Hochschuldidaktik anzuwenden, um Studierenden durch aktives Lernen ein erfolgreiches Studium zu ermöglichen. Im Bereich der quantitativen Ausbildung bedeutet dies, dass Studierende mit grundlegenden Analyseverfahren vertraut gemacht werden und diese in eigenen Studien anwenden können. Zudem besteht im digitalen Zeitalter die Herausforderung für Lehrende darin, aus einer breiten, heterogenen und sich ändernden Menge an modernen Analyseverfahren und Datenarten für Sozialwissenschaften relevante Ansätze zu identifizieren. Diese Ansätze sollen Studierenden dann mit adäquaten E-Lernmaterialien vorgestellt und an sozialwissenschaftlichen Beispielen verdeutlicht werden. Gerade hier sind es die Ansätze der Open Educational Resources und der Open Science, die einen vertieften Austausch auch im Fach Politikwissenschaft einfordern und ermöglichen.

In unserem Beitrag schlagen wir vor, dass Formate der Methodenlehre überdacht werden. Klassische Vorlesungen in Kombination mit Tutorien vermitteln oftmals wenig nachhaltiges Wissen und begeistern Studierende zu wenig für quantitative Forschung. Sie ermöglichen es nicht, dass Studierende einfache Analysen selbstständig umsetzen können, und wirken zu oft abschreckend. Durch das Einbeziehen von praktischen Ansätzen werden Studierende mehr motiviert und begeistert. Durch die Etablierung des Konzepts „Hilfe zur Selbsthilfe“ und die Handreichung konkreter Weiterbildungs- und Selbsthilfewerkzeuge werden Studierende in die Lage versetzt, auch nach dem Studienabschluss wieder zu den Lernmaterialien zurückzukehren. Sie haben idealerweise gelernt, wo sie sich weiterbilden können. Ein Studium vermittelt die Grundlagen und Fähigkeit zum wissenschaftlichen Arbeiten und bereitet den Weg zum lebenslangen Lernen, welches zunehmend mit digitalen Angeboten absolviert wird.

Unser Vorschlag richtet sich an Lehrende. Wir wollen darauf hinweisen, methodische und thematische Seminarkonzepte stärker zu verknüpfen und vermehrt 
aktuelle Entwicklungen der Datenwissenschaft in die Lehre einzubeziehen. Zentrale Vorteile hierdurch sind die gesteigerte Motivation und das Ansprechen von Studierenden. Wir wollen Lehrende dazu ermutigen E-Lernmodule umfangreicher bei der Seminargestaltung einzubeziehen. Gerade im Zusammenhang mit Programmierfähigkeiten gibt es hier eine Bandbreite von Möglichkeiten, welche den Studierenden ein geleitetes und individuelles Lernen daheim ermöglichen. Die universitäre Lehre schafft hier den Rahmen und vernetzt die Studierenden in Präsenzveranstaltungen und im digitalen Raum.

Die Erfahrung aus dem digitalen Sommersemester 2020 in der Coronakrise ermöglicht eine klare Bestandsaufnahme. Lehrende konnten mit hohem Arbeitspensum und persönlichem Engagement Lehrveranstaltungen in digitaler Form umsetzen. Digitale Lehre in der Politikwissenschaft ist möglich, wie es diese besondere Erfahrung verdeutlicht. Die Universitäten in Deutschland und die Fachdisziplin waren allerdings unzureichend auf eine großflächige Umstellung hin zu digitaler Lehre vorbereitet. Es fehlte an verlässlicher Infrastruktur, Erfahrung im E-Lernen und an bewährten digitalen Lernmaterialien. Die nun vorliegenden Erfahrungen zu nutzen, in dem heterogenen Umfeld erfolgreiche Modelle zu identifizieren und noch konsequenter Lehre immer auch digital abzubilden, ist die Herausforderung der kommenden Jahre. Wir hoffen, dass unser Beitrag dabei im Bereich der Methodenausbildung als Motivation und Anregung dienen kann.

Danksagung Der Aufsatz ist eine Ausarbeitung unseres Vortrages auf der Jahrestagung „Vielfalt und Weitblick in der politikwissenschaftlichen Hochschullehre" des Arbeitskreises Hochschullehre der Deutschen Vereinigung für Politikwissenschaft (DVPW) an der Westfälischen Wilhelms-Universität Münster 2019. Wir möchten uns besonders bei Kai-Uwe Schnapp für die umfangreichen Hinweise und Anregungen zur Überarbeitung des Aufsatzes bedanken.

Funding Open Access funding enabled and organized by Projekt DEAL.

Open Access Dieser Artikel wird unter der Creative Commons Namensnennung 4.0 International Lizenz veröffentlicht, welche die Nutzung, Vervielfältigung, Bearbeitung, Verbreitung und Wiedergabe in jeglichem Medium und Format erlaubt, sofern Sie den/die ursprünglichen Autor(en) und die Quelle ordnungsgemäß nennen, einen Link zur Creative Commons Lizenz beifügen und angeben, ob Änderungen vorgenommen wurden.

Die in diesem Artikel enthaltenen Bilder und sonstiges Drittmaterial unterliegen ebenfalls der genannten Creative Commons Lizenz, sofern sich aus der Abbildungslegende nichts anderes ergibt. Sofern das betreffende Material nicht unter der genannten Creative Commons Lizenz steht und die betreffende Handlung nicht nach gesetzlichen Vorschriften erlaubt ist, ist für die oben aufgeführten Weiterverwendungen des Materials die Einwilligung des jeweiligen Rechteinhabers einzuholen.

Weitere Details zur Lizenz entnehmen Sie bitte der Lizenzinformation auf http://creativecommons.org/ licenses/by/4.0/deed.de.

\section{Literatur}

Agresti, Alan, und Barbara Finlay. 2014. Statistical methods for the social sciences. Harlow: Pearson.

Barberá, Pablo. 2015. Birds of the same feather tweet together: Bayesian ideal point estimation using twitter data. Political Analysis 23(1):76-91.

Blätte, Andreas, Joachim Behnke, Kai-Uwe Schnapp, und Claudius Wagemann. 2018. Computational Social Science: Die Analyse von Big Data. Baden-Baden: Nomos. 
Dietrich, Bryce J., Ryan D. Enos, und Maya Sen. 2019. Emotional Arousal Predicts Voting on the U.S. Supreme Court. Political Analysis 27(2):237-243.

Diez, David M., Mine Çetinkaya-Rundel, und Christopher D. Barr. 2019. OpenIntro Statistics. https:// www.openintro.org/book/os. Zugegriffen: 20. März 2020.

Dreiack, Stefanie, Sarah Hartkopf, und Malte Cordes. 2016. Methodenvermittlung in der Politikwissenschaft: Ein Werkstattbericht aus dem PoliMethA-Projekt. Zeitschrift für Politikwissenschaft 26(4):491-499.

Engel, Uwe. 2002. Praxisrelevanz der Methodenausbildung (Sozialwissenschaftliche Tagungsberichte, 5). Informationszentrum Sozialwissenschaften. https://nbn-resolving.org/urn:nbn:de:0168-ssoar263414. Zugegriffen: 20. März 2020.

Goerres, Achim, Caroline Kärger, und Daniel Lambach. 2015. Aktives Lernen in der Massenveranstaltung: Flipped-Classroom-Lehre als Alternative zur klassischen Vorlesung in der Politikwissenschaft. Zeitschrift für Politikwissenschaft 25(1):135-152.

Imai, Kosuke. 2018. Quantitative social science: an introduction. Princeton: Princeton University Press.

Kittel, Bernhard. 2009. Eine Disziplin auf der Suche nach Wissenschaftlichkeit: Entwicklung und Stand der Methoden in der deutschen Politikwissenschaft. Politische Vierteljahresschrift 50(3):577-603.

Lewthwaite, Sarah, und Melanie Nind. 2016. Teaching Research Methods in the Social Sciences: Expert Perspectives on Pedagogy and Practice. British Journal of Educational Studies 64(4):413-430.

Munzert, Simon. 2014. Big Data in der Forschung! Big Data in der Lehre? Ein Vorschlag zur Erweiterung der bestehenden Methodenausbildung. Zeitschrift für Politikwissenschaft 24(1-2):205-220.

Munzert, Simon. 2018. Auf dem Weg zu einer fundierten Softwareausbildung in der Politikwissenschaft. In Computational social science: die analyse von big data, Hrsg. Andreas Blätte, Joachim Behnke, Kai-Uwe Schnapp, und Claudius Wagemann, 379-402. Baden-Baden: Nomos.

Pournas, Evangelos. 2017. Cross-disciplinary Higher Education of Data Science: Beyond the Computer Science Student. Data Science 1(1-2):101-117.

Proksch, Sven-Oliver, und Jonathan B. Slapin. 2014. The politics of parliamentary debate. Cambridge: Cambridge University Press.

Salganik, Matthew J. 2018. Bit by Bit Social Research in the Digital Age. Princeton: Princeton University Press.

Schnapp, Kai-Uwe, und Andreas Blätte. 2018. Epistemologische, methodische und politische Herausforderungen von Big Data. In Computational Social Science: Die Analyse von Big Data, Hrsg. A. Blätte, J. Behnke, K.-U. Schnapp, und C. Wagemann, 27-54. Baden-Baden: Nomos.

Schnapp, Kai-Uwe, Nathalie Behnke, und Joachim Behnke. 2004. Methodenausbildung in der Politikwissenschaft - Oder: Wie aus dem Aschenputtel eine Prinzessin werden kann. In Politikwissenschaft. Rundbrief der Deutschen Vereinigung für Politikwissenschaft, Herbst 2004, Bd. 131, 158-167.

Wickham, Hadley, und Garrett Grolemund. 2016. R for Data Science. Sebastopol: O'Reilly Media.

Wooldridge, Jeffrey. 2015. Introductory Econometrics: A Modern Approach. Boston: Cengage Learning.

Zwick, Markus. 2016. Statistikausbildung in Zeiten von Big Data. AStA Wirtschafts- und Sozialstatistisches Archiv 10(2-3):127-139. 